\title{
PLASMA ECHOES AT UPPER HYBRID RESONANCE*
}

\author{
L. O. Bauer, F. A. Blum, † and R. W. Gould \\ California Institute of Technology, Pasadena, California
}

(Received 14 December 1967)

\begin{abstract}
We report experimental observation of a new plasma echo at upper hybrid resonance and present a simple theoretical model which exhibits some major features of the results.
\end{abstract}

Echoes from a plasma stimulated by multiple excitation pulses were first reported by Hill and Kaplan' in 1965 (cyclotron echoes). Their experiments involved excitation of a plasma in a strong, inhomogeneous magnetic field by pulses whose center frequency was near the electron-cyclotron frequency. Although other mechanisms have been discussed, ${ }^{2}$ a model consisting of a collection of independent electrons gyrating in a nonuniform magnetic field subject to energy-dependent collisions ${ }^{3}$ is believed to give an adequate description of the effect if the plasma density is low enough. More recently, Gould and Blum ${ }^{4}$ discussed theoretically another plasma echo involving upper hybrid plasma oscillations. The upper hybrid echo $^{5}$ is associated with the macroscopic charge and current density fluctuations (at the upper hybrid frequency, $\omega_{h}{ }^{2}=\omega_{c}{ }^{2}+\omega_{p}{ }^{2}$ ) of a plasma column with an inhomogeneous electron-density profile. Thus, collective effects play a dominant role in the formation of this echo. This Letter is a preliminary report on the experimental observation of this new plasma echo and a simple theoretical model which exhibits some salient features of the experimental results.

The plasmas studied experimentally were rare-gas, afterglow discharges created by an rf pulse. They were contained in a glass cylinder of $1.8 \mathrm{~cm}$ i.d. and about $1 \mathrm{~m}$ in length, aligned coaxially with the magnetic field $(\vec{B})$ of a solenoid. The glass tube was inserted through and perpendicular to the narrow walls of $S$-band waveguide so that the configuration for the exciting microwaves was $\overrightarrow{\mathrm{E}} \perp \overrightarrow{\mathrm{k}} \perp \overrightarrow{\mathrm{B}}$, where $\overrightarrow{\mathrm{E}}$ is the electric field and $\overrightarrow{\mathrm{k}}$ is the propagation vector of the signal. The plasma and waveguide formed one of the side arms of a balanced microwave bridge system in which a magic tee was used. In contrast to studies of cyclotron echoes, ${ }^{1,3}$ we studied the upper hybrid echo with $\vec{B}$ homogeneous to one part in $10^{4}$ over the volume of plasma excited by the microwaves. All experimental data reported represent crystal rectifier detected signals.
Seeking knowledge of the normal-mode spectrum of the plasma, we were led to studies of its cw response. ${ }^{6}$ The upper solid curves in Fig. 1 show the cw reflection from a neon plasma for three afterglow times, $T_{a}$. In these experiments the incident signal frequency $\omega / 2 \pi$ $(3.0 \mathrm{Gc} / \mathrm{sec})$ was held constant while the magnetic field, i.e., $\omega_{c}$, the electron cyclotron frequency, was varied. The values of $\omega_{c} / \omega$ are accurate to within $\pm 0.2 \%$. These data show clearly the presence of collective phenomena. The sharp peak near $\omega_{c} / \omega=1$ and the broad peak at lower values are characteristic of all experiments performed. The peak in the scat-

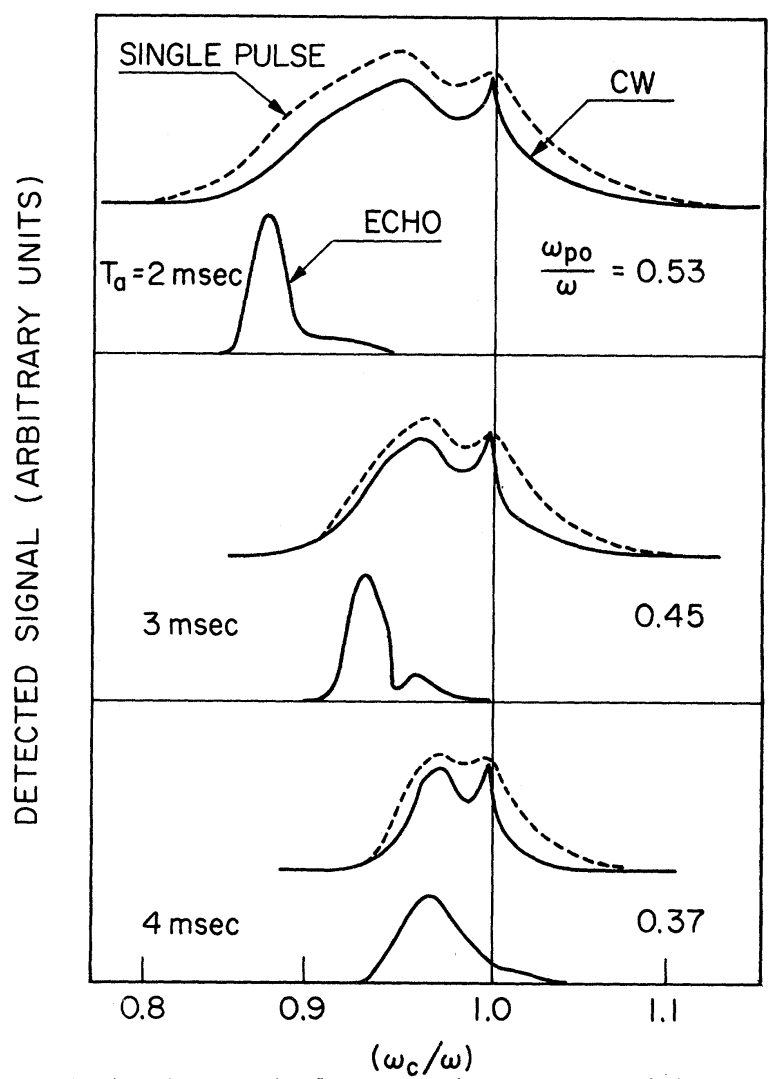

FIG. 1. Composite diagram of experimental data on cw reflection, single-pulse reflection, and echo spectra as a function of $\omega_{c} / \omega$ for a neon afterglow plasma at a pressure of $35 \mu \mathrm{Hg}$. The parameter $T_{a}$ is the time after the end of the breakdown pulse, i.e., the afterglow time. 
tering that significantly shifts and broadens at high electron densities has the appearance of a common feature of reflection, emission, and absorption spectra of cyclotron-resonance experiments reported by other observers. ${ }^{7,8}$ This feature is attributed to the upper hybrid resonance of a cold, inhomogeneous plasma. ${ }^{9}$ In fact, the onset of significant scattering at low values of $\omega_{c} / \omega$ has proved to be a good measure of the maximum electron density of nonuniform plasma columns. ${ }^{8}$ At the onset point on the $\omega_{c} / \omega$ axis the incident signal frequency is equal to the maximum upper hybrid frequency of the plasma, i.e., $\omega^{2}=\omega_{h 0^{2}}=\omega_{c}^{2}+\omega_{p 0}{ }^{2}$, where $\omega_{p 0}$ is the maximum local plasma frequency. This interpretation is the source of the estimates of $\omega_{p 0} / \omega$ given in Fig. 1.

The dashed curves of Fig. 1 demonstrate that the response of the plasma to a high power, very short pulse such as that used in echo experiments is essentially identical with the observed cw scattering. These data are displays of the maximum amplitude of the reflection from the same neon plasma of a 20-nsec microwave pulse of $0.5-\mathrm{W}$ peak power as a function of $\omega_{c} / \omega$. Thus the high-power pulses seem to couple to the same normal modes of the plasma that are observed in low-power cw experiments.

Finally, we come to the echo and its correlation with the $\mathrm{cw}$ and short-pulse spectra. The echoes were stimulated by two pulses separated by a time $\tau$. The pulses were each identical to the one used in the short-pulse reflection experiment. The echoes consist of short bursts of radiation emitted by the plasma at times $t=n \tau, n=1,2, \cdots$, after the second applied pulse. The lower solid curves in Fig. 1 are magnetic field spectra of the first echo $(n=1$, $\tau=100$ nsec), i.e., the dependence of the echo amplitude on $\omega_{c} / \omega$. At $T_{a}=2 \mathrm{msec}$ the echo is strongest at a point which is shifted $13 \%$ (about five times its spectral half-width) from the condition for free electron cyclotron resonance, $\omega_{c} / \omega=1$. Although the data presented are for neon, similar results have been obtained using argon. This is the first observation of echoes under conditions far from those for free electron cyclotron resonance and near what the $\mathrm{cw}$ experiments mark as the maximum upper hybrid frequency of the plasma.

In order to account qualitatively for the general features of the data of Fig. 1, we consider a one-dimensional inhomogeneous cold plas- ma slab $b^{4}$ of thickness $2 a$ situated in a uniform magnetic field $\vec{B}$ which is parallel to the slab faces [see Fig. 2(a)]. The plasma is placed between two parallel conducting plates separated by a distance $2 l$. The steady-state electron density is assumed to depend on $x$ in such a manner that $\omega_{p}{ }^{2}(x)=\omega_{p 0^{2}}\left(1-x^{2} / a^{2}\right)$. Collisions are neglected and the ions are assumed fixed. Simulating the conditions of the experiment, we place a short section of this plasma slab in a parallel-plate transmission line which has a matched generator at one end and is terminated in its characteristic impendance $Z_{0}$ at the other end as shown in Fig. 2(a). We neglect fringing field effects in the short section. The dependence on $\omega_{c} / \omega$ of the reflection coefficient seen at the generator on this transmission line will yield the theoretical equivalent of the experimental $\mathrm{cw}$ curves of Fig. 1. Since in the experiment the lateral dimensions of the plasma are small compared with the wavelength, we replace the plasma of Fig. 2(a) which is distributed along the transmission line by a simple lumped-element equivalent circuit as shown in Fig. 2(b). The circuit element $C_{1}$ is the capacitance of the vacuum between the plasma and the conducting plates, and is given by $C_{1}=\epsilon_{0} A / 2(l-a)$, where $\epsilon_{0}$ is the permittivity of free space and $A$ is the lateral area of the capacitor-slab system. The negative capaci-

(a)

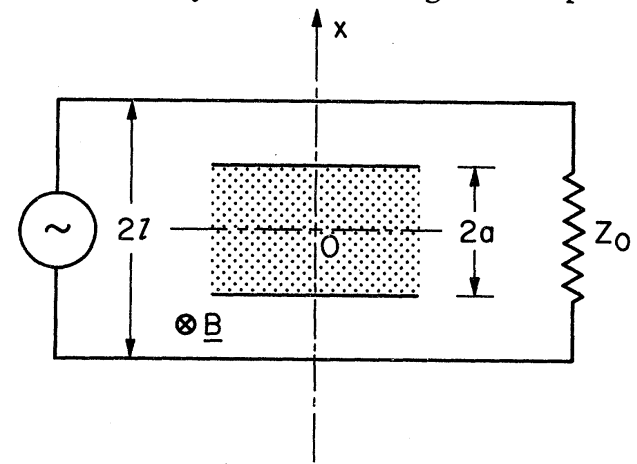

(b)

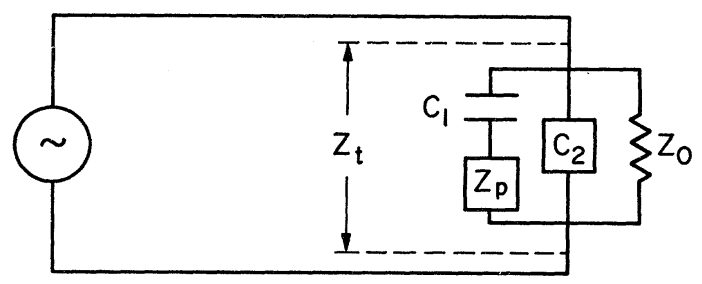

FIG. 2. (a) Diagram showing geometry of the plasma slab and parallel-plate transmission line which comprise the theoretical model. (b) Lumped-element equivalent circuit of the plasma-slab, transmissionline model. 
tance $C_{2}$ results from the equivalent circuit approximation and is equal to $-\epsilon_{0} A / 2 l$. In the linear approximation the impedance of the plas ma slab is given by ${ }^{10}$

$$
Z_{p}=\frac{\left(\omega_{c}^{2}-\omega^{2}\right)}{i \omega \epsilon_{0} A} \int_{-a}^{a} \frac{d x}{\omega_{c}^{2}+\omega_{p}^{2}(x)-\omega^{2}} .
$$

Defining $Z_{t}$ to be the total impedance appearing between the two terminals of the equivalent circuit, one has the complex reflection coefficient measured at the generator, $r=\left(Z_{t}-Z_{0}\right) /$ $\left(Z_{t}+Z_{0}\right)$.

In Fig. 3 (upper curves) we plot $|r|^{2}$ vs $\left(\omega_{c} \mid\right.$ $\omega)$ for several values of the parameter $\omega_{p 0} / \omega$. We have taken $R \equiv Z_{0} l / \omega \epsilon_{0} A=5$ and $l / a=2$ as reasonable estimates, since these parameters do not have exact experimental equivalents. Roughly, $R$ sets the over-all scale of the amount of reflection, while $l / a$ controls the relative height of the two peaks. The values chosen yield results which compare favorably with the experimental data; for example, the max-

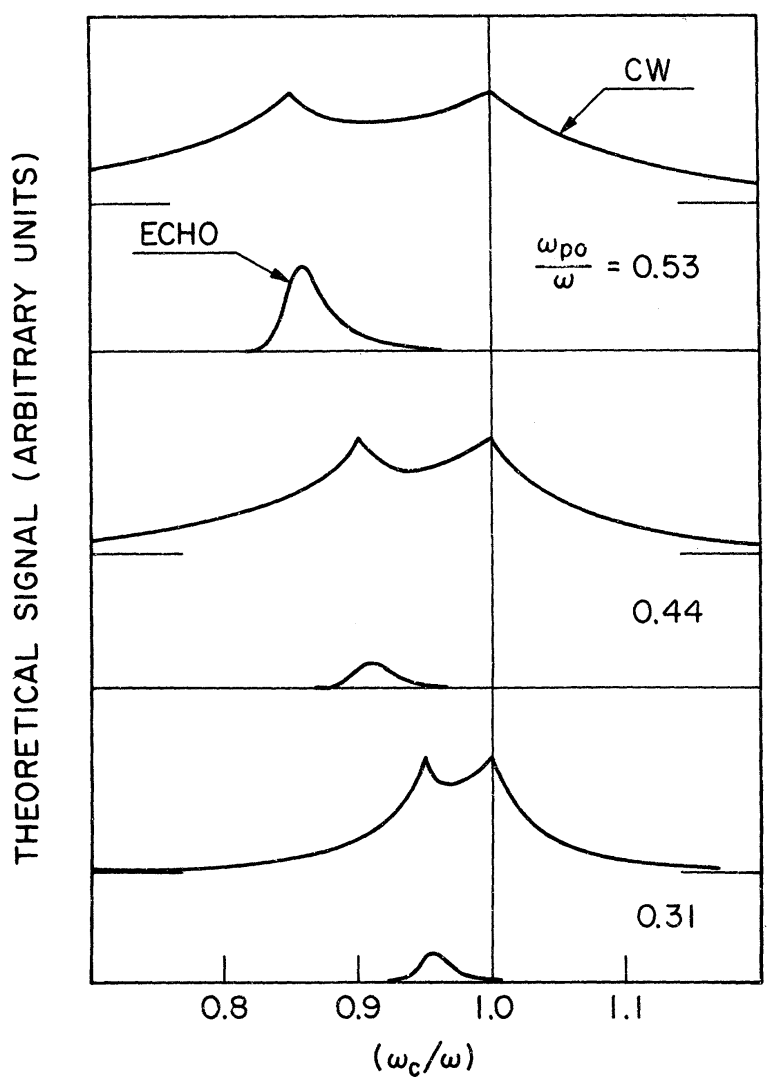

FIG. 3. Theoretical equivalent of Fig. 1. The upper curves $\left(|r|^{2}\right)$ represent the cw reflection, while the lower curves $\left(|E|^{2}\right)$ are the loci of the calculated echo amplitudes $\left(\omega_{p 0} / \omega\right.$ appears as a parameter). imum values of $|r|^{2}$ calculated are approximately the same as those observed experimentally $(\approx 0.5)$. One peak in $|r|^{2}$ occurs at $\omega_{c} / \omega=1$, while the other occurs at the maximum upper hybrid frequency of the slab, $\omega_{h 0}{ }^{2}=\omega_{c}{ }^{2}+\omega_{p 0}{ }^{2}$. The two peaks are a manifestation of the fact that $\left|Z_{p}\right|$ is very high near $\omega=\omega_{h 0}$ and very low near $\omega=\omega_{c}$. The quantitative difference between theory and experiment on the exact position of the upper hybrid peak is one shortcoming of the adopted model.

We have shown previously ${ }^{4}$ that the cold-plasma-slab model has the qualitative features requisite of two-pulse echo systems: the collection of normal modes being the local upper hybrid oscillations and the nonlinearity being furnished by the spatial gradients in $\omega_{p}$. The application of two short pulses results in pulsed echo responses in the voltage across the slab. In order to compute the echo magnetic field spectrum, we must modify previous plasma echo theories to include the limited spectral width of the exciting pulses. For a weak echo theory this modification results in the following expression for the relative peak voltage of the first echo:

$$
\begin{aligned}
E \propto \int_{-a}^{a} d x \frac{\omega_{p}^{2}}{\omega_{h}{ }^{4}}\left\{\left[\omega_{p}^{2}\right]^{\prime \prime}\right. & \left.5\left[\frac{\left(\omega_{p}{ }^{2}\right)^{\prime}}{\omega_{h}}\right]^{2}\right\}\left|f\left(\omega-\omega_{h}\right)\right|^{3},
\end{aligned}
$$

where $f$ is the Fourier transform of one of the exciting pulses and a prime denotes a derivative with respect to $x$. The two pulses are taken to have identical amplitudes and Gaussian shapes of width $\Delta \omega / \omega=0.04$, a close approximation to the experimental conditions. The lower curves of Fig. 3 are plots of $E^{2}$ vs $\omega_{c} / \omega$ computed using Eq. (2). As with the experimental results, the theoretical calculations show the echo to be strongly peaked near the maximum upper hybrid frequency of the plasma. Also, the widths of the theoretical and experimental echo spectra are comparable.

The general features of the theoretical curves in Fig. 3 are not unique to the collisionless model which yields energy-dependent normalmode frequencies. One could include an energy-dependent phenomenological collision frequency supplying both relaxation and another nonlinear effect without qualitatively affecting the results. In either case, the echo is strong- 
ly peaked near the maximum upper hybrid frequency because of the high density of normal modes there and not because of the nature of the nonlinearity.

In the low density limit $\left(\omega_{p} \ll \omega_{c}\right)$ both the upper hybrid echo and the cyclotron echo occur near $\omega=\omega_{c}$. Therefore, the independentparticle theories which have been used rather universally in the interpretation of cyclotron echo experiments ${ }^{2,3}$ cannot be valid in general unless the spread in upper hybrid frequencies due to the spatial dependence of $\omega_{p}$ is considerably less than the spread due to the magnetic field inhomogeneity. ${ }^{11}$ That is, the experiments must satisfy the condition $\omega_{p 0} 0^{2} / 2 \omega_{c} 0^{2}$ $\ll \Delta \omega_{c} / \omega_{c} 0$, where $\Delta \omega_{c}$ is the spread in cyclotron frequencies and $\omega_{c 0}$ is the value of the local cyclotron frequency at the position where the plasma density is the greatest, i.e., where $\omega_{p}=\omega_{p 0}$. For typical laboratory experiments ${ }^{1,3}$ this condition places a significant limitation on the maximum electron density $\left(\approx 10^{9}-10^{10}\right.$ $\mathrm{cm}^{-3}$ ) for which the echo can be correctly understood on the basis of independent particle theories. The competition between these two sources for the spread in upper hybrid frequencies has been studied. ${ }^{11}$

Although the simple plasma model presented gives results strikingly similar to the experimental observations, it should be taken as a first approximation. An appropriate hot plasma theory ${ }^{12}$ would also be expected to yield a set of normal modes whose frequencies are intimately related to the nonuniform electron density profile and the associated upper hybrid frequencies. However, a hot plasma theory, particularly a nonlinear one, would prove considerably more difficult. Also, our theory ignores the problem of coupling to the upper hybrid oscillations. ${ }^{13}$ Kaplan, Hill, and Wong ${ }^{14}$ failed to find echoes near upper hybrid resonance in a high-density cesium plasma. As they discussed, from the point of view of coldplasma theory, this fact may be the result of a failure to couple effectively (due to an evanescent layer) to the region of high normalmode density near the maximum upper hybrid frequency. Other experiments ${ }^{15}$ have produced echoes that are strongest at frequencies between the electron cyclotron frequency and the maximum upper hybrid frequency. Again, this is perhaps a manifestation of the efficiency of coupling to the upper hybrid oscillations. The experiments discussed in this paper would be expected to couple more efficiently to the upper hybrid oscillations, since the wavelength is longer compared with the scale length of the plasma density gradients than that in the other experiments.

The authors are indebted to R. S. Harp, R. M. Hill, D. E. Kaplan, and A. Y. Wong for interesting discussions and communication of their work during the course of this research. In addition, we benefited from stimulating conversations with F. L. Hinton.

\footnotetext{
*Research supported by the U. S. Office of Naval Research under Contract No. Nonr 220(50).

$\dagger$ Howard Hughes Predoctoral Fellow.

${ }^{1}$ R. M. Hill and D. E. Kaplan, Phys. Rev. Letters 14, 1062 (1965).

${ }^{2}$ R. W. Gould, Phys. Letters 19, 477 (1965); W. H. Kegel and R. W. Gould, Phys. Letters 19, 531 (1965); G. F. Herrmann and R. F. Whitmer, Phys. Rev. 143, 122 (1966); W. H. Kegel, Phys. Letters 23, 317 (1966), and Plasma Phys. 9, 23, 339 (1967); L. O. Bauer, R. W. Gould, and W. H. Kegel, Bull. Am. Phys. Soc. 12, 756 (1967).

${ }^{3}$ D. E. Kaplan and R. M. Hill, Bull. Am. Phys. Soc. 11, 496, 538 (1966); G. F. Herrmann, R. M. Hill, and D. E. Kaplan, Phys. Rev. 156, 118 (1967); F. W. Crawford and R. S. Harp, Phys. Letters 21, 292 (1966), and J. Appl. Phys. 37, 4405 (1966); D. E. Kaplan, R. M. Hill, and A. Y. Wong, Phys. Letters 22, 585 (1966); R. S. Harp, R. L. Bruce, and F. W. Crawford, J. Appl. Phys. 38 , 3385 (1967).

${ }^{4}$ R. W. Gould and F. A. Blum, in Proceedings of the Eighth International Conference on Phenomena in Ionized Gases (Springer-Verlag, Vienna, Austria, 1967), p. 405.

${ }^{5}$ The possibility of plasma echoes due to effects of upper hybrid resonance was first suggested by G. F. Herrmann and R. F. Whittmer [Phys. Rev. 143, 122 (1966)].

${ }^{6}$ Since relatively low-power cw microwaves were found to heat the plasma, experiments on the magnetic field dependence of the reflections from the plasma using a wide $(\approx 1 \mu \mathrm{sec})$, low-power $(-30 \mathrm{dBm})$ pulse were performed in the bridge system. These widepulse experiments yield results essentially identical with those of a low-power cw experiment, if the spectral width of the pulse is narrow compared with the normal mode spectrum width of the plasma.

${ }^{7}$ G. Bekefi, J. D. Coccoli, E. B. Hooper, and S. J. Buchsbaum, Phys. Rev. Letters $\underline{9}, 6$ (1962); S. J. Buchsbaum and A. Hasegawa, Phys. Rev. Letters 12, 685 (1964), and Phys. Rev. 143, 303 (1966); S. Tanaka, H. Kubo, and K. Mitani, J. Phys. Soc. Japan 20, 462 (1965).

${ }^{8}$ H. J. Schmitt, G. Meltz, and P. J. Freyheit, Phys. Rev. 139, A1432 (1965); C. D. Lustig, Phys. Rev. 139, A63 (1965); S. Tanaka, J. Phys. Soc. Japan 21, 1804 (1966).
} 
${ }^{9}$ S. J. Buchsbaum, Bull. Am. Phys. Soc. $\underline{7}, 151$ (1962); S. J. Buchsbaum, L. Mower, and S. C. Brown, Phys。 Fluids $\underline{3}, 806$ (1960).

${ }^{10}$ When $\omega$ lies in the band of upper hybrid frequencies, the integrand of Eq. (1) has a simple pole for some value of $x(|x| \leqslant a)$. The inclusion of electron collisions removes this singularity from the path of integration. Taking the limit of zero collision frequency tells one how to integrate around the pole. This limit is equivalent to the Dirac formulation of such integrals and is responsible for giving $Z_{p}$ a real part.

${ }^{11}$ R. S. Harp, R. L. Bruce, and F. W. Crawford, J. Appl. Phys. 38, 3385 (1967).
${ }^{12}$ See, for example, G. A. Pearson, Phys. Fluids 9 , 2454 (1966); H. L. Frisch and G. A. Pearson, Phys. Fluids $\underline{9}, 2464$ (1966).

${ }^{13}$ For a discussion of this problem, see G. Bekefi, Radiation Processes in Plasmas (John Wiley \& Sons, Inc., New York, 1966), p. 223.

${ }^{14}$ D. E. Kaplan, R. M. Hill, and A. Y. Wong, Phys. Letters 22, 585 (1966).

${ }^{15}$ L. O. Bauer, F. A. Blum, and R. W. Gould, in the Ninth Annual Meeting of the Division of Plasma Physics of the American Physical Society, Austin, Texas, 8-11 November 1967 (unpublished); R. M. Hill and D. E. Kaplan, private communication.

\title{
COLLISION-INDUCED LIGHT SCATTERING
}

\author{
Howard B. Levine and George Birnbaum \\ Science Center/Aerospace and Systems Group, \\ North American Rockwell Corporation, Thousand Oaks, California 91360
}

(Received 22 January 1968)

We consider here a new type of light scattering in gases and liquids which arises as a result of molecular collisions. Because of the distortion of the electronic structure during collision, the polarizability of colliding molecules is not the sum of the individual polarizabilities. For binary collisions, this incremental polarizability scatters light into a broad frequency band whose width is controlled by the duration of collision and whose intensity varies as the square of the density. Such scattering can be viewed as Rayleigh scattering by the collision-induced polarizability, or as Raman scattering involving a change in the translational state of the relative kinetic energy of a pair of colliding molecules. Our purpose is to derive a simple analytic expression for the spectrum of scattered light which provides a framework for discussing the nature of the effect, to consider what systems are most suitable for observation, and to indicate experiments in which the effect may have been seen although not clearly recognized as such.

Following our previous work in collisioninduced absorption, ${ }^{1}$ we (1) consider binary collisions, (2) assume straight line paths, and (3) assume the variation of the incremental polarizability with interatomic separation to be of the form

$$
\begin{aligned}
& \alpha(r)=A_{1} \exp \left(-\gamma_{1}{ }^{2} r^{2}\right), \\
& \beta(r)=A_{2}\left(\gamma_{2} r\right)^{2} \exp \left(-\gamma_{2}{ }^{2} r^{2}\right),
\end{aligned}
$$

where $\alpha(r)$ and $\beta(r)$ are, respectively, the spher- ical and anisotropic parts of the induced polarizability, defined in the usual way in terms of $\alpha_{\|}(r)$ and $\alpha_{\perp}(r)$. These equations exhibit the proper asymptotic behavior: (1) $\alpha(r) \rightarrow 0$ as $r \rightarrow \infty$, and $\alpha(r)$ assumes a finite limit as $r \rightarrow 0$; and (2) $\beta(r) \rightarrow 0$ as $r \rightarrow \infty$, and $\beta(r) \rightarrow 0$ as $r \rightarrow 0$ by reason of the spherical symmetry of the united atom. ${ }^{2}$

Assuming a plane-polarized incident light beam with an electric vector, $\overrightarrow{\mathrm{E}}=\overrightarrow{\mathrm{i}} E_{0} \cos \left[\omega_{0}\right.$ $\left.\times\left(t-z c^{-1}\right)\right]$, we apply classical radiation theory $^{3}$ to compute the energy spectrum of the light scattered by the induced dipole moments, which are proportional to $\alpha(r)$ and $\beta(r)$, during a single collision. The assumptions stated above mean that the energy pulses dominantly have a Gaussian envelope in time. Multiplication by the collision rate $^{4}$ and integration over the relative velocity and impact parameter then yields the power spectrum of the scattered light. For the noble gases, it is found to be the sum of two contributions:

$$
\begin{aligned}
& \frac{d^{2} J_{\kappa}^{(1)}}{d \Omega d \omega}=\frac{2 A_{1}{ }^{2} N_{0}{ }^{2}}{c^{4} V_{0}{ }^{2}}\left(\frac{\pi k T}{2 m}\right)^{3 / 2} \\
& \quad \times x_{1}{ }^{4} P_{\kappa}^{(2)}\left(\left|x_{2}-x_{02}\right|\right)
\end{aligned}
$$

due to the spherical term $\alpha(r)$ and

$$
\begin{aligned}
\frac{d^{2} J_{\kappa}^{(2)}}{d \Omega d \omega}= & \frac{2 A_{2}{ }^{2} N_{0}^{2}}{15 c^{4} V_{0}^{2}}\left(\frac{\pi k T}{2 m}\right)^{3 / 2} \\
& \times x_{2}{ }^{4} P_{\kappa}^{(2)}\left(\left|x_{2}-x_{02}\right|\right)
\end{aligned}
$$

